This paper is published in the open archive of Mid Sweden University

DIVA http://miun.diva-portal.org

with permission of the publisher

Citation for the peer-reviewed published paper:

Pettersson G, Wågberg L, Höglund H. The use of polyelectrolyte multilayers of cationic starch and $\mathrm{CMC}$ to enhance strength properties of papers formed from mixtures of unbleached chemical pulp and CTMP-II : Part II: Influnence of addition strategy, fibre treatment and fibre type. ; Nordic Pulp \& Paper Research Journal. 2006;21(1):122-128.

URL to article at publishers site:

http://dx.doi.org/10.3183/NPPRJ-2006-21-01-p122-128 


\title{
The use of polyelectrolyte multilayers of cationic starch and CMC to enhance strength properties of papers formed from mixtures of unbleached chemical pulp and CTMP. Part II Influence of addition strategy, fibre treatment and fibre type
}

\author{
Gunilla Pettersson and Hans Höglund, Mid Sweden University, Sundsvall, Lars Wågberg, Royal Institute of Technology, KTH, Stockholm, Sweden
}

KEYWORDS: Adsorption, Interactions, Mechanical properties, Multilayer, Polyelectrolytes, Polymers, Surfaces

\begin{abstract}
SUMMARY: Polyelectrolyte multilayers (PEM) consisting of cationic starch and anionic carboxymethylcellulose (CMC) have been applied to different pulp fibres in order to enhance the outof-plane sheet strength properties of a typical packaging board furnish. An unbleached softwood chemical pulp was treated with multilayers consisting of two layers of cationic starch and one layer of $\mathrm{CMC}$, and then mixed with different mechanical and chemimechanical pulps. Hand sheets were prepared with the aid of the Rapid Köthen sheet former from stocks consisting of $20 \%$ treated chemical pulp and $80 \%$ mechanical or chemimechanical pulp, which was either PGW from spruce, HTCTMP from spruce or birch, or a standard spruce CTMP. Multilayer treatment significantly improved Scott Bond values and in some cases improved the tensile index, with the achieved effects being significantly larger than the effects of applying starch alone. Positive effects were obtained by treating only $20 \%$ of the furnish, showing a very high efficiency of the adsorbed multilayers.

Compared to earlier work, one important finding was that the PEM treatment should preferably be applied only to the chemical pulp and not on the entire stock. It was possible to increase the out-of-plane strength properties, measured as Scott Bond values, with just a very small increase in density of the sheets. Multilayer treatment of the chemical pulp improved the joint strength between the fibres while maintaining the high bulk of the sheets prepared from the stiff mechanical and chemimechanical fibres.
\end{abstract}

\section{ADDRESSES OF THE AUTHORS: Gunilla Pettersson (gunilla.pettersson@miun.se) and Hans Höglund, (hans.hoglund@miun.se): Mid Sweden University, Department of Natural Sciences, Fibre Science Communication Network, FSCN, SE-851 70 Sundsvall, Sweden Lars Wågberg (wagberg@polymer.kth.se): Royal Institute of Technology, KTH, Department of Fibre and Polymer Technology, Division of Fibre Technology, SE-100 44 Stockholm, Sweden. Corresponding author: Gunilla Pettersson}

Pulping processes separate the fibres from wood and making them suitable for papermaking. Separating the fibres from each other without causing significant damage occurs only if the lignin is either dissolved, as is the case in chemical pulping, or efficiently softened, as in mechanical and chemimechanical pulping. All types of pulp fibres must be mechanically treated before the fibres become suitable for papermaking. In chemical pulping this occurs as a separate process in the paper mill, while this happens during the pulping process itself for mechanical and chemimechanical pulping. Most mechanical pulps are used in printing paper grades, with the ideal mechanical pulp forming a sheet of paper with high opacity, brightness, bulk and smoothness, and satisfactory strength properties.

Mechanical and chemimechanical pulps are also used in paperboard packaging materials (Swan and Otto 1985, Tomas 1997, Höglund 1997, Höglund and Wilhelmsson 1993), which require the pulps to give a high bending stiffness at certain internal bond strengths. Carton boards are usually manufactured with a multi-ply stratified structure. A high bending stiffness in stratified carton boards is obtained by combining a middle ply having high bulk (low density) with outer layers having a high tensile stiffness. This suggests that mechanical and chemimechanical pulps are preferable for use in the middle ply to create a bulky structure (Fellers et al.1983, Hägglund and Höglund, 1992) while chemical pulps are used in the outer plies (Höglund and Wilhelmsson 1993, Fineman 1985). Mechanical and chemimechanical pulps should preferably have a high content of long fibres with relatively high stiffness. The benefits of combining mechanical and chemimechanical pulps with chemical treatments, i.e., polyelectrolyte multilayers, are detailed in this report, ultimately focusing on improving Scott Bond properties without significantly increasing the sheet density.

In this respect PolyElectrolyte Multilayers (PEM) can be used to strengthen the joint between fibres via a consecutive treatment of the fibres with a cationic and an anionic polyelectrolyte respectively (Wågberg and Forsberg 1998, Pettersson et al 2005); this procedure can in principle be repeated for a third, fourth, and fifth layer. Since papers made from mechanical pulps often have a low in-plane and out-of-plane strength, PEM technology would be very advantageous for preparing ductile fibre joints in order to optimise paper quality. Furthermore, PEMs offer an alternative for producing new graphic paper products with superior runnability and could also be ideal to utilise the inherent strength and stiffness of mechanical pulp fibres for packaging papers.

Regarding dry strength additives in general in papermaking, cationic starch is the most commonly used additive (Reynolds, 1980, Roberts et al., 1986, Wågberg and Björklund, 1993). Due to the combination of a relatively low cost and a rather high efficiency in improving paper properties, it has been very difficult to beat cationic starch on a cost/performance basis. However, cationic starch gives at best a $10-15 \%$ strength increase when adsorbed as a single layer, which is often too little for 
certain applications. It is rather well established that there is a correlation between the adsorbed amount and the strength-enhancing effect of the additive and therefore different approaches, apart from changing the charge of the strength-enhancing additive, have been used to increase the adsorption. Combinations of cationic and anionic polyelectrolytes (Stratton 1989, Stratton and Colson 1993) and cationic polyelectrolytes, inorganic complexes and anionic polyelectrolytes (Strazdins 1980) have been utilised in order to increase the adsorbed amount of the strength additives. It was shown (Stratton 1989) that the combination of cationic and anionic polyelectrolytes in the wet end of papermaking was much more efficient than a treatment of the dry paper, and furthermore it was also shown that the additives were made efficient via an increase in the fibre/fibre contact. Different types of combinations of polyelectrolytes were also tested, and it was demonstrated (Stratton and Colson 1993) that ionic linkages between chemicals were as efficient as, or even more efficient than, polyelectrolytes capable of forming covalent linkages. As was mentioned earlier, it is possible to get higher strength properties with the relatively new idea of polyelectrolyte multilayering. The idea of applying PEM technology as paper strength additives was first brought forward by Wågberg and Forsberg (Wågberg and Forsberg 1998). The first practical application aimed at adsorbing high amounts of cationic PAE (polyamideamine epichlorohydrine), a common wet strength additive, to improve wet strength in tissue paper by combining the PAE treatment with a carboxymethylcellulose CMC treatment (Sandberg and Andreasson 2000). Later work along these lines has also shown that the structure of the multilayers (Eriksson et al., 2005a, Notley et al., 2005) as well as the chemical structure of the used chemicals (Eriksson et al. 2005b) have a large influence on the strength-enhancing properties. PEMs of cationic amylose and anionic potato starch were also shown to be much more efficient than conventional PEMs of conventional cationic and anionic potato starch (Eriksson et al. 2005b). In Notley et al. (2005) it was furthermore shown that a high mobility of the polyelectrolyte multilayer was important to create a strong fibre/fibre joint. This report focuses on maximising the dry strength, and therefore cationic starch and anionic $\mathrm{CMC}$, which are known to contribute to dry strength (Sandberg and Andreasson 2000), are selected as the polyelectrolytes.

\section{Materials and Methods}

\section{Materials}

Pulps. An unbleached softwood kraft pulp was used in furnishes together with different mechanical and chemimechanical pulps. Four different mechanical and chemimechanical pulps were tested: an unbleached spruce CTMP from Skoghall, Sweden; bleached HT (High Temperature)-CTMP from both spruce and birch from Östrand, Sweden; and a bleached spruce pressure groundwood (PGW) from Ingerois, Finland. These mechanical pulps span a broad range of properties, namely regarding the long fibre content and the ability to form strong fibre joints. Pulps manufactured according to the HT-CTMP technique can be produced with low energy consumption at a very high CSF level (Höglund 2002) and low shive contents.

The unbleached kraft pulp and pressure groundwood pulp (PGW) were delivered at a solids content of $4 \%$, filtered on a funnel with a plastic filter, and washed with distilled water before being filtered again and refrigerated. The unbleached chemical pulp was cooked to kappa number 22 and was a mixture of spruce and pine. The standard CTMP was delivered at a solid content of $30 \%$ pulp and stored as received and it was washed in distilled water before use. The HT-CTMPs were delivered dry and washed in distilled water before use.

Polyelectrolytes. Potassium polyvinyl sulphate (KPVS) from Wako Pure Chem. Ltd., Japan, and 3.6-ionene, Polybrene, from Sigma Aldrich were used in the polyelectrolyte titration to determine the fibre charge. Both polyelectrolytes were delivered as powders and used without further purification.

The surface charge determination was made with a high molecular mass polyDADMAC, Alcofix 109, supplied by Ciba Specialty Chemicals. Ultrafiltration was used to remove the low molecular mass fraction in order to make the polyelectrolyte suitable for surface charge determinations. The theoretical charge density of the polyDADMAC was 6.19 meq./g. The molecular mass of the filtered polymer was $1.2 \cdot 10^{6}$, as determined by size exclusion chromatography (Swerin and Wågberg 1994).

Potato starch, CS, supplied by Lyckeby Stärkelsen, Sweden, with a cationic degree of substitution of 0.065 , corresponding to a charge density of $0.38 \mathrm{meq} / \mathrm{g}$, was used. The starch was laboratory cooked by heating a $1 \mathrm{~g} / \mathrm{l}$ starch slurry to $95{ }^{\circ} \mathrm{C}$, maintaining this temperature for $30 \mathrm{~min}$, and allowing the starch solution to cool down under ambient conditions. Fresh solutions of starch were prepared every day in order to avoid the influence of starch degradation.

CMC (Cekol $50000 \mathrm{G}$ ) was obtained from Noviant, Finland. The degree of substitution in the product was 0.8 and the molecular weight was about 900000 . The CMC, which contained a high purity ( $>99.5 \% \mathrm{CMC})$, was dispersed in deionised water at a concentration of $1 \mathrm{~g} / \mathrm{l}$ and allowed to dissolve $12 \mathrm{~h}$ before use.

Other chemicals. Hydrochloric acid, sodium hydroxide, and sodium chloride used in the investigation were all of analytical grade.

\section{Methods}

Total charge determination. Conductometric titration (Katz, et al. 1984) was used to determine the total charge on the unfractionated pulp. $0.5 \mathrm{~g}$ of fibres was diluted in deionised water to a total volume of $500 \mathrm{ml}$. The starting $\mathrm{pH}$ was set to 3.8 with $0.1 \mathrm{M} \mathrm{HCl} .10 \mathrm{ml}$ of $0.01 \mathrm{M} \mathrm{NaCl}$ was added to minimise the uneven distribution of ions between the fibre phase and the external water phase, caused by the Donnan equilibrium in the fibre/water system.

Surface charge determination. The surface charge of the cellulosic fibres was determined by the polyDADMAC 
adsorption method (Wågberg, Ödberg, and GladNordmark 1989). As the high molecular polyDADMAC cannot penetrate the external surface, the amount of polyDADMAC adsorbed can be taken as a measure of the fibre surface charge.

Adsorption measurements. Polyelectrolyte titration was used to establish the adsorption isotherms for the three layers. The anionic CMC solutions were titrated with polybrene, and the cationic starch solutions were titrated with KPVS to the isoelectric end point. The charge in the solution was thereafter determined from the amount of either the cationic or anionic charges added per litre sample. The adsorbed amount was calculated by comparing the amount of non-adsorbed polyelectrolyte in the solution with the amount of polyelectrolyte initially added.

Adsorption experiments were performed at a fibre concentration of $5 \mathrm{~g} / 1$ in $0.01 \mathrm{M} \mathrm{NaCl}$ at $\mathrm{pH}$ 6. Different amounts of either starch or $\mathrm{CMC}$, depending on which layer was being adsorbed, were added to a series of at least five pulp samples diluted to $100 \mathrm{ml}$. The samples were stirred for $10 \mathrm{~min}$ to reach adsorption equilibrium and the suspensions were then filtered with a Büchner funnel fitted with a \#3 Munktell filter. The clear filtrate was collected and the amount of polyelectrolyte remaining in solution was titrated using a polyelectrolyte titrator from Mütek, GmbH, Germany (Bley and Kastner 1992).

Excess polyelectrolyte must be removed before further layers can be adsorbed, and the non-adsorbed polyelectrolyte from the previous layer was filtered off using a Büchner funnel. The filter pad was also carefully washed with a small amount of distilled water.

Determination of the amount of starch in the prepared papers. The amount of starch was determined by an enzymatic method. With this method, the starch molecules are enzymatically degraded to glucose units, which are then quantified by HPLC measurement (Wågberg and Björklund, 1993).

Fractionation of pulp and fibre treatment. The unbleached softwood kraft pulps were fractionated using tap water following the SCAN M6: 69 method .The pulp that did not pass the 200-mesh wires was collected and used as the long fibre fraction.

The unbleached softwood kraft pulp was circulation refined at $4 \%$ consistency in a Voith Sulzer LR1 laboratory refiner with conical refiner fillings having the following parameters: $3 \mathrm{~mm}$ bar width, 60 degree cutting angle, flow rate $100 \mathrm{l} / \mathrm{min}$, rotational speed $2000 \mathrm{rpm}$, constant specific edge load of $3.5 \mathrm{Ws} / \mathrm{m}$. Fibres with a degree of beating of 22 SR were chosen for the majority of the experiments.

Before fibre preparation, the CTMP pulps were hot disintegrated according to SCAN M10: 77 at $85{ }^{\circ} \mathrm{C}$ in distilled water. The unbleached softwood chemical pulp was reslushed in distilled water for 30000 revolutions according to SCAN C: 1865. After reslushing, the fibres were combined at a mixing ratio of $80 \%$ PGW or CTMP and $20 \%$ unbleached softwood chemical pulp. The final concentration was $5 \mathrm{~g} / \mathrm{l}$ in a solution of $0.01 \mathrm{M} \mathrm{NaCl}$ at $\mathrm{pH} 6$. Before combining the chemical pulp with the CTMP or PGW, the chemical pulp was treated with the polyelectro- lytes in different steps. Cationic starch was first adsorbed, followed by a treatment with $\mathrm{CMC}$, and then again with starch. Each polyelectrolyte was allowed to adsorb for 10 min while being stirred. After multilayer treatment and mixing of the different pulps, the fibre suspension was transferred to the sheet preparation equipment. Sheets were made with 1,2 , and 3 multilayers at $\mathrm{pH} 6$, corresponding to typical conditions for the production of liquid packaging board. From a theoretical point of view, adsorbing at $\mathrm{pH} 8$ might have been "safer" as all carboxyl groups are dissociated, but $\mathrm{pH} 6$ was chosen to investigate the applicability of the PEM concept to industrial production of liquid packaging board.

Sheet preparation and paper testing. Sheets were made on a Rapid Köthen sheet former from Paper Testing Instruments (PTI), Pettenbach, Austria. Sheets with a grammage of $150 \mathrm{~g} / \mathrm{m}^{2}$ were formed after vigorous aeration of the fibre suspension just before sheet preparation. The sheets were then pressed at $100 \mathrm{kPa}$ and dried under restrained conditions at $94{ }^{\circ} \mathrm{C}$. The ISO/DIS 5269-2 method was followed for sheet preparation. Tensile testing was conducted according to SCAN P: 67 and grammage, thickness and density were evaluated according to SCAN C 28:76. Scott Bond testing was conducted according to Tappi 833 pm-94.

It is well known that cationic starch might induce severe fibre flocculation that might give negative results on the strength of the papers containing the starch (Roberts et al., 1986). Therefore the formation of the sheets was carefully checked by ocular inspection and no change in formation of the sheets following starch addition could be detected. This was probably due to the vigorous stirring of the furnish in the Rapid Köthen equipment after the starch addition and before the sheet formation.

\section{Results and Discussion}

\section{Charge determination of the different pulps}

In order to characterise the ability of the different fibres to interact with the polyelectrolytes, fibres were subjected to both conductometric titration and surface charge determination by the polyelectrolyte adsorption method. The results from these measurements are shown in Table 1. The total charge of the chemical pulp is around $100 \mu \mathrm{eq} / \mathrm{g}$, although the beaten fibres have a somewhat higher charge. This is a bit unexpected and indicates that beating creates new surfaces in the fibre wall that are not initially available for the $\mathrm{OH}-$ ions in conductometric titration. The surface charge determinations of the chemical pulps show the large influence of the fines material on the available surface charge of the pulp. As the degree of beating increases, the total surface charge of the furnish changes from 8.3 to $20.6 \mu \mathrm{eq} / \mathrm{g}$, corresponding to a relative change from 8.7 to $18.8 \%$ of the total charge. The surface charge of the long fibre fraction changes from $7 \mu \mathrm{eq} / \mathrm{g}$ to $10.4 \mathrm{meq} / \mathrm{g}$, which corresponds to a change from $8.1 \%$ to $10.3 \%$ of the total charge. Thus it becomes possible to adsorb more chemicals to the chemical pulp as the beating increases, as there is a direct correlation between surface charge and 
adsorption of most types of polymeric paper chemicals.

The bleached mechanical pulps, specifically the HTCTMP and the PGW, have a higher charge compared with the unbleached CTMP. Table 1 also shows that the ratio of surface charge to the total charge varies between $9.5 \%$ and $13.9 \%$ for these pulps.

In order to clearly determine the influence of the strategy of only adsorbing PEMs to the chemical pulp, the treated chemical pulps were washed before being mixed with the mechanical pulps. If not washed, the excess of polyelectrolyte would have been indirectly adsorbed to the mechanical pulp, making the interpretation of the results difficult. In future investigations it will naturally be important to determine the influence of dissolved and colloidal material on the starch adsorption and, as shown earlier (Wågberg and Kolar 1996), it is important to balance the charge of the starch and the type of dissolved and colloidal material in the furnish. This was considered to be beyond the scope of the present investigation.

\section{Adsorption of polyelectrolytes and influence of beating on adsorption}

It has previously been shown (Pettersson et al 2005) that it is possible to adsorb anionic CMC in a second adsorbed layer to a fibre with a pre-adsorbed layer of cationic starch. The adsorption measurements were used to determine an appropriate addition of starch and CMC in the different layers.

These types of measurements were also repeated for pulps with different degrees of beating, and the results are summarised in Table 2. These values were described and discussed earlier in Pettersson et al (2005) and are in the present work included to facilitate interpretation of the results from the strength measurements. To summarise, the data in Table 2 show that a combination of beating and PEM treatment could be a useful way of reaching high levels of polyelectrolyte adsorption to unbleached chemical fibres. However, only the pulp beaten to 22 SR was chosen for further studies because it has a very high adsorption capacity. A higher degree of beating would probably result in an excessive densification of the

Table 1. Summary of the charge characteristics of the various pulps and the respective ratio of surface to total charge.

\begin{tabular}{lccc}
\hline Material & $\begin{array}{c}\text { Total charge } \\
\mu \mathrm{eq} / \mathrm{g}\end{array}$ & $\begin{array}{c}\text { Surface charge } \\
\mu \mathrm{eq} / \mathrm{g}\end{array}$ & $\begin{array}{c}\text { Relative charge } \\
\%\end{array}$ \\
\hline $\begin{array}{l}\text { Unbleached softwood } \\
\text { chemical pulp without fines }\end{array}$ & & & \\
- Unbeaten & 86.0 & 7.0 & 8.1 \\
- SR 22 & 96.9 & 9.8 & 10.1 \\
- SR 33 & 101.0 & 10.4 & 10.3 \\
Unbleached softwood & & & \\
chemical pulp with fines & & & \\
- Unbeaten & 95.7 & 8.3 & 8.7 \\
- SR 22 & 99.2 & 18.4 & 18.5 \\
- SR 33 & 110.0 & 20.6 & 18.7 \\
CTMP & 99.5 & 13.8 & 13.9 \\
HT-CTMP birch & 159.2 & 15.2 & 9.5 \\
HT-CTMP spruce & 144.0 & 14.2 & 9.9 \\
PGW & 135.0 & 16.1 & 11.9 \\
& & & \\
\hline
\end{tabular}

Table 2. The adsorbed amount of polyelectrolyte in layers 1-3 on the unbleached chemical pulp beaten to different degrees.

\begin{tabular}{lccc}
\hline Number of layer & $\begin{array}{c}\text { Unbeaten } \\
\text { sulphate } \\
(\mathbf{m g} / \mathbf{g})\end{array}$ & $\begin{array}{c}\text { Beaten } \\
\text { sulphate SR 22 } \\
(\mathbf{m g} / \mathbf{g})\end{array}$ & $\begin{array}{c}\text { Beaten } \\
\text { sulphate SR 33 } \\
(\mathbf{m g} / \mathbf{g})\end{array}$ \\
\hline 1 (starch) & 8.0 & 22.0 & 31.0 \\
2 (CMC) & 16.0 & 39.0 & 45.0 \\
3 (starch) & 24.0 & 65.0 & 74.0 \\
\hline
\end{tabular}

sheets, which would be a disadvantage when considering bending stiffness of the prepared papers.

\section{Strength of sheets prepared with Starch/CMC multilayer treatment}

In the previously published results (Pettersson, Wågberg, and Höglund 2005), regarding the treatment of mixtures of spruce CTMP and chemical pulps, it was found that there were large advantages to using a PEM treatment compared to increasing the amount of sulphate pulp in the furnish regarding in-plane and out of plane strength properties. However, despite these positive results it was clear that PEM treatment increased the sheet density. To counteract this effect, PEMs are only adsorbed to the chemical pulp before mixing it with the CTMP. In order to be comparable with the previous study, the same ratios of mixing between the CTMP and the chemical pulp were used. The results from these experiments are shown in Figs 1-3, where the full lines correspond to the entire furnish being treated, and the broken lines correspond to the treatment of only the unbleached sulphate pulp.

The response in Scott Bond values and the tensile energy absorption values from treating just the chemical pulp is comparable to treating the entire furnish. For the $80 / 20$ case the effect of the PEM addition only to the sulphate pulp seems to be a bit smaller than the effect of the PEM addition to the entire furnish. However, taking the difference in densification between the two addition strategies into consideration, it is clear that the achieved affect of the PEM addition is comparable. The improvements in Scott Bond values can also be achieved with a smaller density increase, which is very important for maintaining a high bending stiffness of the board at a particular Scott Bond value. Another very important result is that the improvement in Scott Bond and tensile energy absorption can be achieved at a much lower chemical addition, since only a smaller fraction of the furnish is treated. It should be noted that the improvement in tensile index is somewhat less than when the whole furnish is treated. Despite achieving significant improvements in Scott Bond and tensile energy absorption already at $20 \%$ chemical pulp in the furnish, other mixtures were also investigated, in order to allow for comparison to the earlier presented PEM treatments (Pettersson et al 2005)

\section{Influence of beating of the chemical pulp}

Since it was sufficient and advantageous to treat only the chemical pulp with the PEMs, it became relevant to investigate how this treatment affects properties of sheets prepared from chemical pulp with different degrees of bea- 


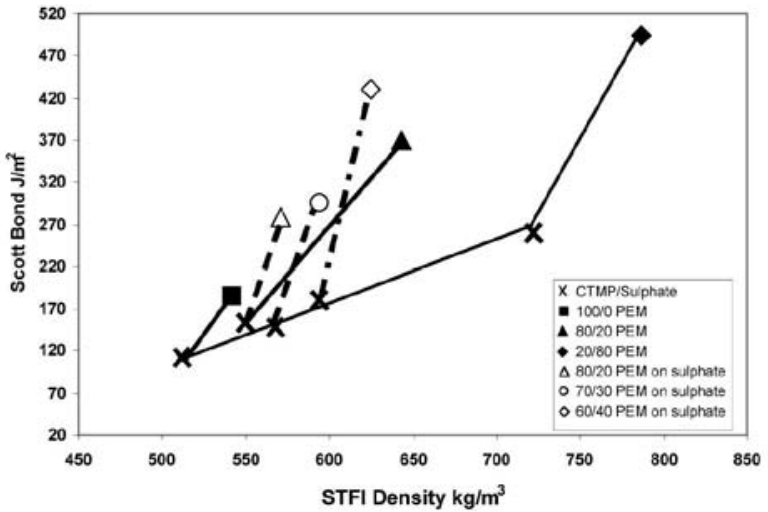

Fig 1. Scott Bond values of sheets made from mixtures of unbleached CTMP and unbleached softwood chemical pulp. The ratio of unbleached chemical pulp was varied from 0 to $80 \%$ (i.e., $100 / 0$ and $20 / 80$ in the figure). The full lines correspond to earlier published results where the PEM treatment was conducted on the entire furnish. The dotted line shows results from experiments where the polyelectrolytes were added only to the unbleached sulphate

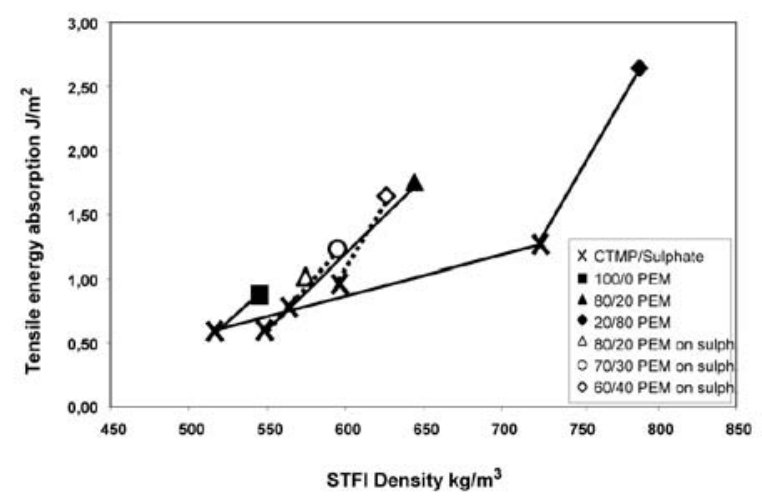

Fig 2.Tensile energy adsorption values for sheets made from unbleached CTMP in mixtures together with unbleached softwood chemical pulp. The amount of unbleached chemical pulp was varied from 0 to $80 \%$ (i.e., 100/0 and 20/80 in the figure) and the full lines correspond to earlier published results where the PEM treatment was conducted on the entire furnish. The dotted line shows results from experiments where the polyelectrolytes were added only to the unbleached sulphate. The PEM treatment consisted of two layers of starch with a CMC layer in between.

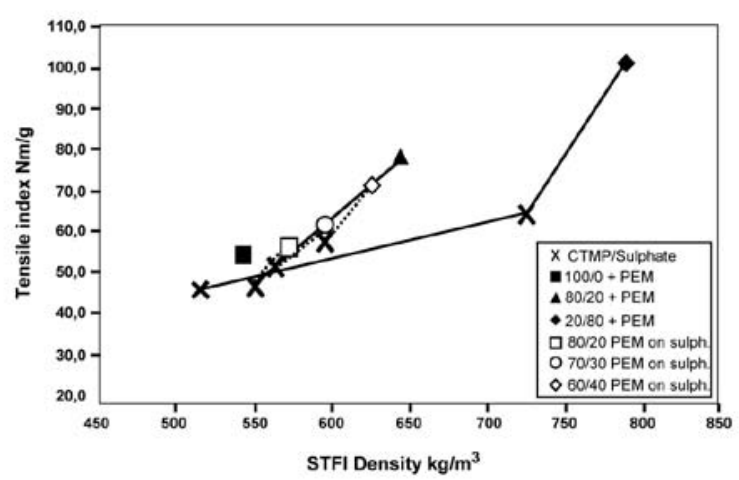

Fig 3. Tensile index values for sheets made from unbleached CTMP in mixtures together with unbleached softwood chemical pulp. The amount of unbleached chemical pulp was varied from 0 to $80 \%$ (i.e., 100/0 and 20/80 in the figure) and the full lines correspond to earlier published results where the PEM treatment was conducted on the entire furnish. The dotted line shows results from experiments where the polyelectrolytes were added only to the unbleached sulphate. The PEM treatment consisted of two layers of starch with a CMC layer in between.

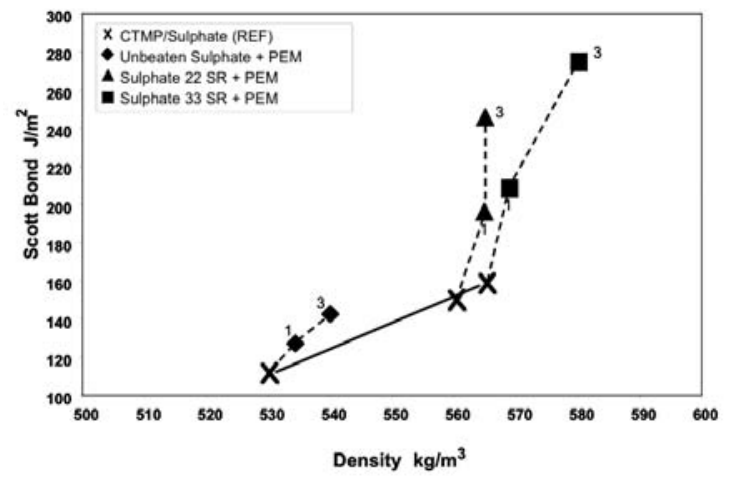

Fig 4. Scott Bond values of sheets from unbleached CTMP and unbleached sulphate pulp at $80 / 20$ mixture at three different degrees of beating. Multilayer treatment was conducted in three layers. Ref=reference no PEM treatment, 1) One layer of starch. 3) Two layers of starch with a CMC layer in between.

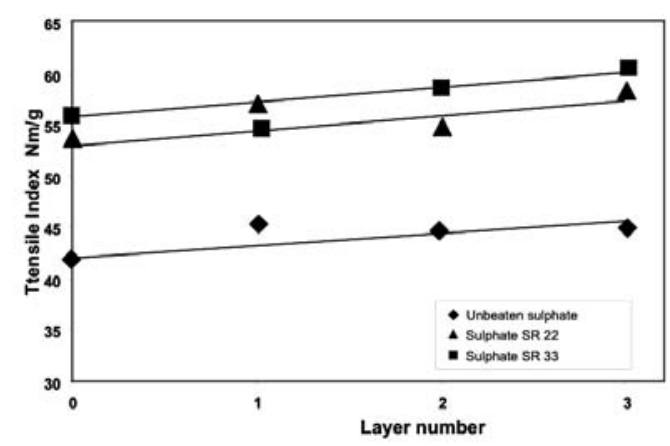

Fig 5. Tensile index values of sheets made from unbleached CTMP from and unbleached sulphate pulp at 80/20 mixtures. The unbleached chemical pulp was beaten to three different degrees and treated with various numbers of polyelectrolyte layers. Ref=reference no PEM treatment, 1) One layer of starch. 3) Two layers of starch with a CMC layer in between.

ting. The effect of beating, seen in Fig 4, shows that PEMs have a better effect on highly beaten pulps. This effect partly depends on a more flexible fibre and larger surface area of the beaten fibres, which increase the contact area in the fibre/fibre joint. The sizeable influence of beating is also linked to the fact that the beaten fibres can adsorb a larger amount of starch and CMC compared to the unbeaten fibres, see Table 2. Beating the pulps without PEM treatment results in a higher sheet density and only a minor increase in Scott Bond, shown by the dotted line in Fig 4. On the other hand, PEM treatment increases the Scott Bond but yields only a small increase in the density, specifically seen for the beaten pulps. Treatment of the unbeaten pulp shows a densification without a large increase in Scott Bond. It should also be noted that adsorption of three layers of polyelectrolytes almost doubles the Scott Bond values for the beaten pulps.

The tensile index measurements show that the tensile properties are improved with an increased degree of beating, as can be seen in Fig 5 for the pulps beaten to 22 SR and $33 \mathrm{SR}$.

A unique trend can be noticed in comparing Figs 4 and 5. Whereas beating of the chemical pulp increases the Scott Bond value only to a minor extent, beating the pulp to 22 SR increases the tensile strength by more than $25 \%$. PEM treatment results in an increase in tensile index of only 
around 5\%. This shows that the mechanisms behind improvements in Scott Bond and tensile strength are quite different for these types of mixtures of chemical pulps and mechanical pulps. Further investigations are needed to resolve this phenomenon.

\section{Influence of PEM treatment on mixtures of unbleached chemical pulps and different types of mechanical and chemimechanical pulps}

It was also of interest to investigate the possibility of achieving even better effects by using a CTMP of higher freeness. High temperature CTMPs from spruce and birch, each having a relatively higher fraction of long fibres than standard CTMP, were tested. For comparison, a SGW pulp with a relatively smaller long fibre fraction was also investigated.

Strength data for sheets made from these pulps are contained in Figs 6 and 7. The results are similar to those presented earlier for a 20/80 mixture of the 22 SR unbleached sulphate pulp, where PEM treatment of only the chemical pulp largely improved the Scott Bond with a small densification of the sheets. It is also clear that the best effect, regarding improvement of Scott Bond values, can be achieved for the birch HT-CTMP. The best effect with the PEM treatment regarding tensile index can be reached for the spruce HT-CTMP. The improvements of strength properties of the PGW are small. However, the PGW is very bulky and the initial Scott Bond values are surprisingly high. The high bulk indicates that the PGW fibres are stiffer than the fibres from the CTMP. The exact reasons for these different effects are not known, but the results clearly indicate that it is possible to produce board structures with totally new and interesting properties from a combination of a suitable mechanical treatment of the chemical pulp, PEM treatment of the chemical pulp, and a CTMP with a large amount of long and stiff fibres.

\section{Conclusion}

A new way of engineering fibre surfaces through PEM treatment has been presented whereby the fibres, i.e., mixtures of chemical and mechanical or chemimechanical pulp, are consecutively treated with cationic starch and anionic CMC. The present results showed that it was possible to achieve large improvements in out-of-plane properties, measured as Scott Bond values, without sheet densification by treating only the chemical pulp used in mixtures with CTMP. Furthermore, it was shown that this improvement was achieved without large improvements of other sheet strength properties, such as tensile index.

The influence of beating of the chemical pulp in combination with PEM treatment also showed that the tensile index was significantly increased with the beating whereas the out-of-plane properties were not changed to the same extent. For the PEM treatment the results were the opposite, i.e. PEM treatment of the beaten pulps resulted in significant improvements in Scott Bond values but only small increases in tensile index.

Different types of mechanical and chemimechanical

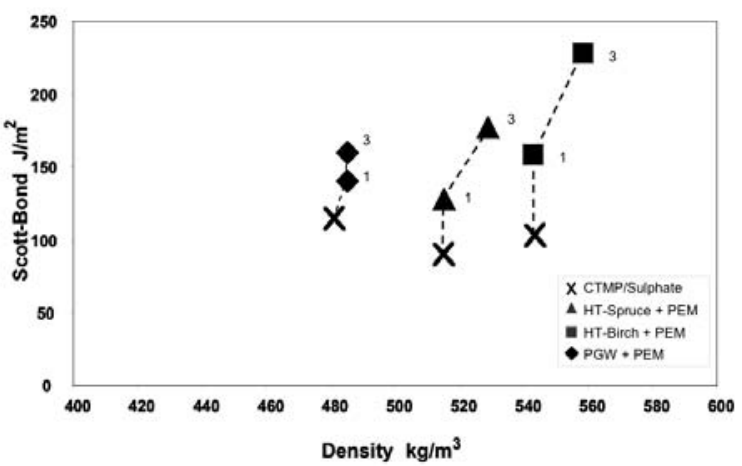

Fig 6. Scott Bond values of sheets from PGW, HT-CTMP from spruce and HTCTMP from birch mixed together with an unbleached chemical pulp beaten to SR 22 at 80/20 mixture. The PEM treatment was conducted in three layers. Ref=reference no PEM treatment, 1) One layer of starch. 3) Two layers of starch with a CMC layer in between.

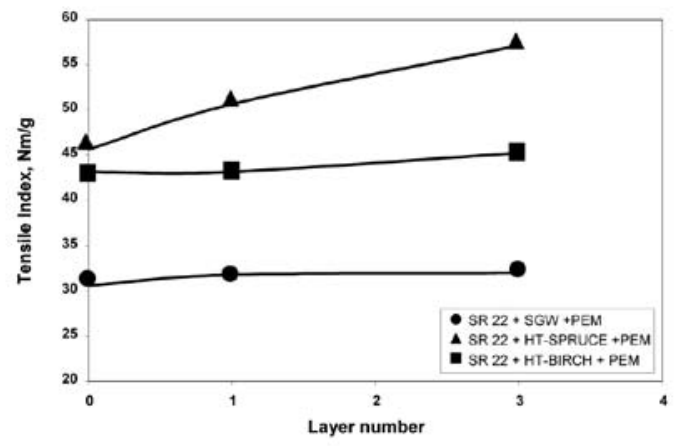

Fig 7. Tensile strength of sheets from PGW, HT-CTMP from spruce and HT-CTMP birch used together with an unbleached chemical pulp beaten to SR 22 at 80/20 mixture. The PEM treatment was conducted in three layers. Ref=reference no PEM treatment, 1) One layer of starch. 3) Two layers of starch with a CMC layer in between.

pulps were tested in combination with the PEM treated chemical pulp and it was found that the concept could also be applied to these types of pulps. Of the different pulps, the High Temperature CTMP from birch showed the largest improvement in out-of-plane properties with the PEM treatment.

It can finally, then, be concluded that the treatment of the fibres with polyelectrolyte multilayers is a new way of functionalising the fibre surfaces, and the applications might range from hygiene products to paperboard since these products often contain mixtures of chemical pulps and mechanical pulps.

\section{Literature}

Aksberg, R. and Ödberg, L. (1990): Adsorption of an anionic polyacrylamide on cellulosic fibres pre-adsorbed cationic polyelectrolytes, Nordic Pulp Paper Res. J. 4, 168.

Bley, L. and Kastner, M. (1992): Paper presented at SPCI-ATICELCA 92, European Pulp and Paper Week: New Available Techniques and Current Trends, Paper Control 19-22 May 1992, Bologna, Italy, 455.

Eriksson, M., Notley, S.M. and Wågberg, L. (2005a): The influence on paper strength properties when building multilayers of weak polyelectrolytes onto wood fibres, Accepted for publication in J. Colloid Interface Sci.

Eriksson, M., Pettersson, G. and Wågberg, L. (2005b): Application of polymeric multilayers of starch onto wood fibres to enhance strength properties of paper, Nordic Pulp Paper Res. J. 20(3), 270.

Fellers, C., de Ruvo, Alf., Htun, M., Carlsson, L., Engman, C. and Lundberg, 
R. (1983): Carton Board, Profitable use of pulps and processes, STFI, Stockholm. Fineman, I. (1985) Let the paper product guide the choice of mechanical pulp, International Mechanical Pulping Conference, Stockholm, Sweden, May 6-10.

Hägglund, T-Å. and Höglund, H. (1992): Recent development of CTMP for absorbent products, The New Nonwoven World, 1(1), 93.

Höglund, H. (1997): Tomorrow's challenges for mechanical pulp, International Mechanical Pulping Conference, Stockholm, Sweden, 9-13 June, 29.

Höglund, H. (2002): Mechanical pulp fibres for new and improved paper grades 7th International Conference on New Available Technologies, Stockholm, Sweden, 4-6 June, 158.

Höglund, H. and Wilhelmsson, K. (1993): The product must determine the choice of wood type in mechanical pulping, International Mechanical Pulping Conference, Oslo, Norway, 15-17 June, 1.

Katz, S., Beatson, R.P. and Scallan, A.M. (1984): The determination of strong and weak acidic groups in sulfite pulps, Svensk Papperstidning, 87(6), R48.

Notley, S.M, Eriksson, M. and Wågberg, L. (2005): Visco-elastic and adhesive properties of adsorbed polyelectrolyte multilayers determined in situ with QCM-D and AFM measurements, Accepted for publication in J. Colloid Interface Sci.

Pettersson, G., Wågberg, L. and Höglund, H. (2006): The use of polyelectrolyte multilayers of cationic starch and CMC to enhance strength properties of papers formed from mixtures of unbleached chemical pulp and CTMP, Nord. Pulp Paper Res J. 21(1) 115-121.

Reynolds, W.F. (1986): Papermakers Conference, Dry strength additives, Tappi Press, Atlanta, 337.

Roberts, J.C., Au, C.0., Clay, G.A. and Lough, C. (1986): The effect of C14labelled cationic and native starches on dry strength and formation, Tappi J. 69 (10), 88.

Sandberg, S. and Andreasson, B. (2000): Swedish Patent Application SE 0001268-2.
Stratton, R.A. (1989): Dependence of sheet properties on the location of adsorbed polymer, Nordic Pulp Paper Res. J. 4(2), 104.

Stratton, R.A. and Colson, N.L. (1993): Fibre wall damage during bond failure, Nordic Pulp Paper Res. J. 8(2), 245.

Strazdins, E. (1980): Neue chemische Erkentnisse zur Optimierung der Trockenfestigkeit von altpapierhaltigen Stoffzusammenstezungen, Papier, $34(10 \mathrm{~A}), \mathrm{V} 49$.

Swan, B. and 0tto, T. (1985): International Mechanical Pulping Conference, Stockholm, Sweden, 6-10 May, 290.

Tomas, H. (1997): International Mechanical Pulping Conference, Stockholm, Sweden, 9-13 June, 411.

Wågberg, L. and Kolar, K. (1996): Adsorption of cationic starch on fibres from mechanical pulps, Berichte der Bunsen-Gesellschaft, 100(6), 984.

Wågberg, L., Ödberg, L. and Glad-Nordmark, G. (1989): Charge determination of porous substrates by polyelectrolyte adsorption, Nordic Pulp Paper Res. J. 4(2), 71.

Wågberg, L. and Björklund, M. (1993): Adsorption of cationic potato starch on cellulosic fibres, Nordic Pulp Paper Res. J. 8(4), 399.

Wågberg, L. and Forsberg, S. (1998): International patent application W0 00/32702.

Swerin, A. and Wågberg, L. (1994): Use of HPLC-methodology to characterize cationic polymers used in the paper industry, Nordic Pulp Paper Res. J. 9(1), 18.

Wågberg, L., Ödberg, L., Lindström, T. and Aksberg, R. (1988): Kinetics of adsorption and ion-exchange reactions during adsorption of cationic polyelectroIytes onto cellulosic fibres, J. Colloid Interface Sci. 123 (1), 287.

Manuscript received August 24, 2005 Accepted December, 2005 